\title{
Suicide Risk among Immigrants and Ethnic Minorities: A Literature Overview
}

\author{
Alberto Forte ${ }^{1}$, Federico Trobia ${ }^{2}$, Flavia Gualtieri ${ }^{2}$, Dorian A. Lamis ${ }^{3}$, Giuseppe Cardamone ${ }^{4}$, \\ Vincenzo Giallonardo ${ }^{5}$, Andrea Fiorillo ${ }^{5}$, Paolo Girardi ${ }^{1}$ and Maurizio Pompili ${ }^{1, *}$ (iD \\ 1 Department of Neurosciences, Mental Health and Sensory Organs, Suicide Prevention Center, Sant'Andrea \\ Hospital, Sapienza University of Rome, 00189 Rome, Italy; alberto.forte@yahoo.it (A.F.); \\ paolo.girardi@uniroma1.it (P.G.) \\ 2 Psychiatry Residency Training Program, Faculty of Medicine and Psychology, Sapienza University of Rome, \\ 00189 Rome, Italy; trobiafed@gmail.com (F.T.); gualtieriflavia@gmail.com (F.G.) \\ 3 Department of Psychiatry \& Behavioral Sciences, Emory University School of Medicine, \\ Atlanta, GA 30303, USA; dalamis@gmail.com \\ 4 Psychiatric Department, Azienda USL Toscana Sud-Est, 53100 Siena, Italy; \\ giuseppe.cardamone@uslsudest.toscana.it \\ 5 Department of Psychiatry, University of Campania Luigi Vanvitelli, 80138 Naples, Italy; \\ enzogiallo86@gmail.com (V.G.); andrea.fiorillo@unicampania.it (A.F.) \\ * Correspondence: maurizio.pompili@uniroma1.it; Tel.: +39-06-3377-5675; Fax: +39-06-3377-5342
}

Received: 1 June 2018; Accepted: 5 July 2018; Published: 8 July 2018

\begin{abstract}
Recent studies have demonstrated that immigrants and ethnic minorities may be at higher risk of suicidal behaviour as compared to the general population. We conducted a literature search to identify studies in English from 1980 to 2017 related to suicide risk among immigrants and ethnic minorities. Six hundred and seventy-eight reports were screened, and 43 articles were included in the qualitative synthesis of the review. Some studies reported lower rates of suicide attempts, while other findings suggested higher rates of suicidal behaviour and deaths among immigrants as compared to the native population. Also, a positive correlation was found between suicidal behaviour and specific countries of origin. Non-European immigrant women were at the highest risk for suicide attempts, a group which included young women of South Asian and black African origin. Risk factors among migrants and ethnic minorities were found to be: language barriers, worrying about family back home, and separation from family. The lack of information on health care system, loss of status, loss of social network, and acculturation were identified as possible triggers for suicidal behaviour. Overall, results suggest that specific migrant populations and ethnic minorities present a higher risk of suicidal behaviour than native populations, as well as a higher risk of death by suicide.
\end{abstract}

Keywords: immigrants; ethnic minorities; suicide; prevention

\section{Introduction}

Recently, a growing need for a better understanding of mental health issues among immigrants and ethnic minorities has emerged worldwide [1-5]. Europe is now facing the largest migration since the Second World War, resulting in new research questions about the extent of the burden of mental health disorders in migrants [1]. Migrants often experience physical and emotional trauma, including being victims of torture. This may be related to their risk of several psychological problems, such as post-traumatic stress disorder (PTSD), mood and anxiety disorders, and panic attacks, with symptoms of sleeplessness, nightmares, and flashbacks.

Several studies have suggested an increased risk of common mental disorders such as depression as well as psychotic disorders in immigrants as compared to native populations [6,7]. A meta-analysis 
suggested that migration could be considered a unique risk factor for severe mental health disorders such as schizophrenia [8]. Many studies also focused on women's mental health, and suggested that immigrant women are at higher risk for postpartum depression than non-immigrant women [9].

Recent research has demonstrated that immigrants may be at a higher risk for suicidal behaviour $[10,11]$. Several authors have also suggested that suicide risk may vary among ethnic minorities [12], and they may have different and more specific risk factors for suicidal behaviour than the general population, such as acculturative stress [13]. As suggested by Wyatt and colleagues [13], acculturation (a process by which subjects acquire the attitudes, values, customs, beliefs, and behaviours of a different culture) may play a role in the development of a suicidal crisis among migrants and ethnic minorities, besides classical psychosocial and psychopathological risk and protective factors [13]. Moreover, the impact of acculturative stress on public health is complex and the underlying theoretical framework, its public health impact, and mental health implications are not yet clear $[14,15]$. To our knowledge, this is the first study providing a literature overview on suicidal behaviour and specific risk factors both in migrants and ethnic minorities. Thus, the aim of the present paper was to provide an overview of the literature on suicide risk among these two populations and explore potential differences between them.

\section{Material and Methods}

\subsection{Search Strategy}

In order to provide a novel and timely systematic review concerning suicidal behaviour among immigrants and ethnic minorities, we used the PRISMA (Preferred Reporting Items for Systematic Reviews and Meta-Analyses) statement for reporting systematic reviews [16]. We conducted a MedLine, Excerpta Medica, PsycLit, PsycInfo, and Index Medicus search to identify all papers and book chapters in English on the main topic for the period from January 1980 to March 2017. We used as specific key words: "suicide" (or suicidal or suicidality), "ethnic minorities" (or ethnicity, ethnic groups, ethnic communities), and "migrants" (or immigrants), including additional references with hand-searched bibliographies of screened articles.

\subsection{Study Selection}

As shown in Figure 1, a total of 998 abstracts were screened through the initial database searches. After duplicates were removed, a total of 678 reports were obtained and full texts were screened with a brief examination of titles and analysis of abstracts. Of these, 77 articles were identified as potentially relevant, and 45 were included in the qualitative synthesis based on the consensus of at least two authors. We gathered data from the initial 77 articles using a template form in Microsoft Excel, according to study characteristics, geographical area of interest, and results.

\subsection{Eligibility Criteria}

Articles that met the following criteria were included: (1) publication in a peer-reviewed journal between 1980 and 2017; (2) publication in English; (3) original study (e.g., not a review); (4) focus on suicidal behaviour; and (5) analysis on immigrants and/or ethnic minority population. The exclusion criteria applied were: failure to report on suicidal behaviour, sampling only from geriatric or paediatric subjects, repeated reports from the same study, and not reporting in English. Thirty-three articles did not fulfil the inclusion criteria, and the remaining 44 were included in the final review. 


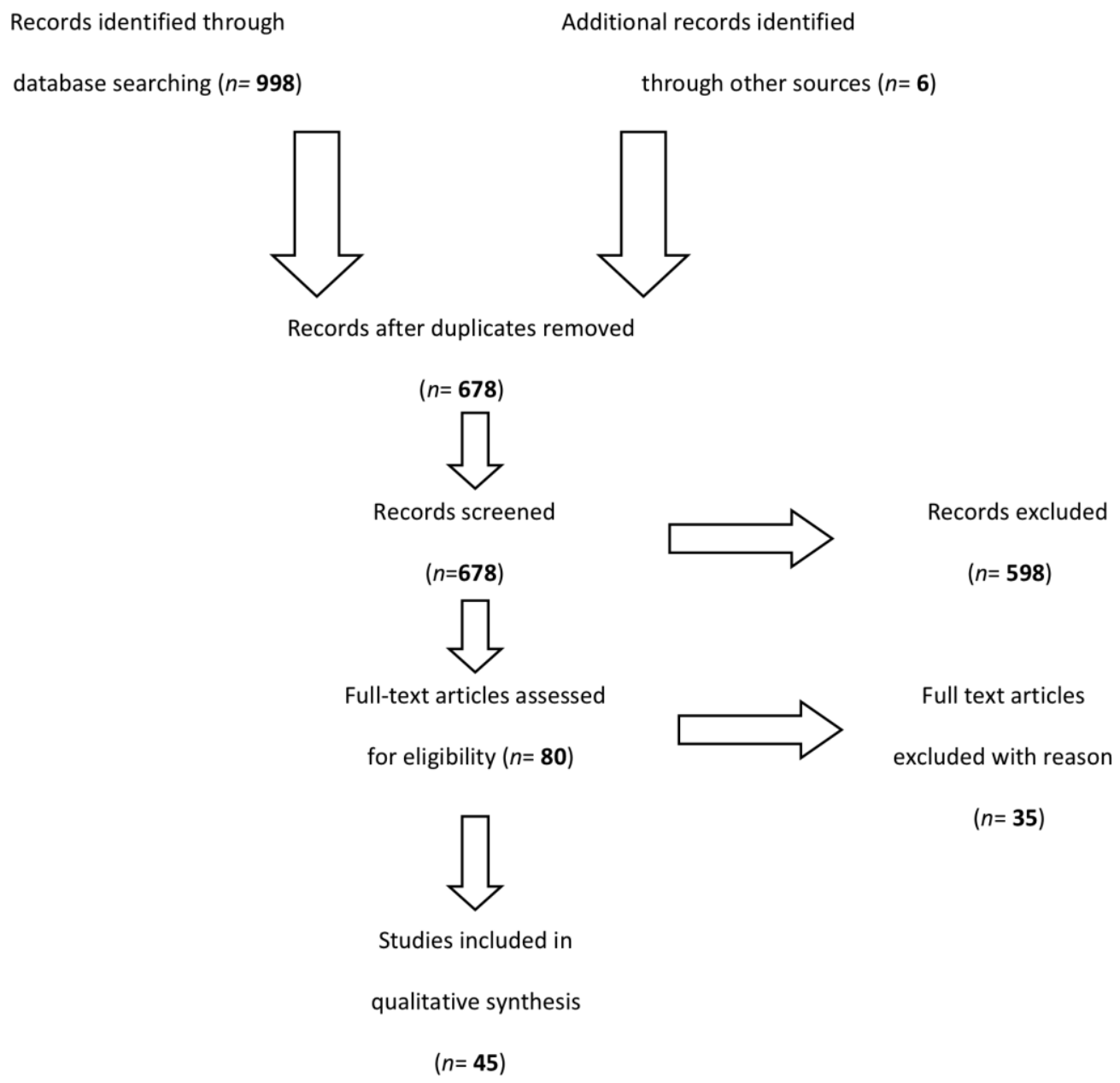

Figure 1. Search strategy and study selection.

\subsection{Study Concept, Quality Assessment and Terminology}

The main aim of this research was to examine suicidal behaviour in immigrants and ethnic minority populations, reviewing the recent literature on this topic in order to identify and understand possible risk factors for suicide and attempted suicide in the investigated population.

A quality assessment was performed as shown in Table 1, rating studies with the following criteria: (I) sample size (not reported 0 points, $<10001$ point, $\geq 10002$ points, $\geq 10,0003$ points); (II) study method ( 1 or 2 points); (III) evidence-based measures assessing suicide or suicide attempts ( 0 or 1 points); and (IV) reliability of data ( 1 or 2 points). The maximum score obtainable for each study was 8 .

To clarify the terminology used in the article, suicidal behaviours refers to both suicide (resulting in a fatal outcome), attempted suicide, and suicidal ideation (thoughts about suicide such as plans and ideas) [17].

With the term migrant (or immigrant), we refer to the definition of the International Organization for Migration (IOM). Although there is no formal legal definition of an international migrant, most experts agree with IOM, which describes a migrant as any person who is moving or has moved across an international border or within a state away from his/her habitual place of residence regardless of: (1) the person's legal status; (2) whether the movement is voluntary or involuntary; (3) what the causes for the movement are; or (4) what the length of the stay is. In addition, the types of migration can be categorized into short-term migration (within 3 and 12 months), and long-term migration (for a duration of one year or more). 
The term refugee refers to the definition of the Statute of the United Nations High Commissioner for Refugees (UNHCR), which delineates a crucial legal difference with respect to the migrant population [18]. Although it is common to see the two terms used incorrectly as synonyms, refugees are specifically all persons who are outside their country of origin for reasons of violence, persecution, or conflict. As a result, they require international protection and are covered by international law.

We found some discrepancies with regards to the use of the term ethnic minority. There is no legal definition of ethnic minorities in international law; however, in Europe the definition is provided by the European Charter for Regional or Minority Languages and by the Framework Convention for the Protection of National Minorities. National (ethnic) minorities can be theoretically (not legally) defined as a group of people living within a national state with the following characteristics: (1) status of a minority among the citizen of that state or representing a smaller group than the rest of the population of the state; (2) distinct culture, language, religion and will to preserve them; (3) not in a dominant position; and (4) long-term presence in the territory [19].

\section{Results}

\subsection{Suicide Attempts and Ideation in Immigrants}

As shown in Table 1, some studies reported that immigrants have lower rates of suicide attempts than the native population, with annual suicide mortality rates of 1.79 per 100,000 among adolescents with migrant status, compared to 3.05 for native-born individuals [20]. Moreover, a significantly decreased risk of depression was also reported: $10.8 \%$ for the migrant group, as compared to $18 \%$ for non-migrant group [21]. Conversely, other studies did not find a significant difference in rates among migrants and natives [22], or found mixed results [23-26]. Taken together, a majority of studies showed higher rates of suicide attempts among immigrants than the native population [27-29]. Several years ago, in Sweden, a higher risk ratio (1.3) was found for attempts in both men and women of foreign-born minorities as compared to the native population [30]. In England, suicide rates in migrants ranged from 15 to $17.4 \%$, which are above the native-born rates [31,32]. Filipino migrants working in home care in Israel reported a prevalence of lifetime suicide attempts of $4.5 \%$ as compared to a rate of $1.4 \%$ in their home country [33]. Similar results were found among ethnic minorities in the Netherlands: Turkish and Surinamese females had a higher risk of attempted suicide, with a rate of $483 / 100,000$ compared to 246/100,000 of Dutch females of the same age [34]. Duldulao and colleagues discovered that first-generation United States-born Asian American women had a significantly higher prevalence of suicidal ideation than their other national counterparts [35]. Overall, data suggested that non-European immigrant women are at the highest risk of suicide attempts [23,32,34], although a positive correlation between suicidal crisis and specific countries of origin was found. A rate of 37.7/1000/year was found for South Asian women as compared to 23.3/1000/year for white women and 23.9/1000/year for black women [36]. Black and Asian minority groups in Europe and the USA were shown to have an increased risk of developing mental health problems as well as a higher risk of associated factors for suicide attempts $[23,36,37]$.

\subsection{Suicide Deaths in Immigrants}

Taken together, results indicated a higher risk of suicide deaths among immigrants and ethnic minorities. Shah and colleagues demonstrated that suicide mortality ratios in foreign males living in England and Wales were generally higher in those of younger age groups (between 20-24 and 50-54 years) born in Eastern European or Caribbean regions. The rates were generally lower in those born in Western Europe in the younger age group, and higher in the age bands of 75-79 and 80-84 years of age. Woman in older age bands (70-74 and 85+ years) and coming from African, Caribbean, and Chinese countries were found to have a higher suicide mortality ratio [38]. Bhui highlighted higher suicide rates among young women of South Asian origin and black African origin, with SMRs (Standardized Mortality Ratios) of 2.8 and 2.7, respectively [39]. 
Table 1. Summary of reports on suicide risk in ethnic minorities and immigrants stratified by region.

\begin{tabular}{|c|c|c|c|c|c|c|c|c|}
\hline Study & Quality Score & Country & Aim and Study Design & Sample & Attempt $N$ & Suicide $N$ & Methods & Main Findings \\
\hline \multicolumn{9}{|l|}{ Ethnic minorities } \\
\hline $\begin{array}{l}\text { O'Keefe et al., } \\
2014 \text { [40] }\end{array}$ & $\begin{array}{l}\text { I:1 } \\
\text { II:1 } \\
\text { III:0 } \\
\text { IV:1 } \\
\text { Total: } 3\end{array}$ & USA & $\begin{array}{l}\text { Investigated American } \\
\text { Indian students' } \\
\text { suicidal ideation and its } \\
\text { predictability. } \\
\text { Observational study. }\end{array}$ & 171 & not reported & not reported & On-line survey. & $\begin{array}{l}\text { Thwarted belongingness and } \\
\text { perceived burdensomeness } \\
\text { predict suicidal ideation in } \\
\text { American Indians. }\end{array}$ \\
\hline $\begin{array}{l}\text { Scheel et al., } \\
2011 \text { [41] }\end{array}$ & $\begin{array}{l}\text { I:1 } \\
\text { II:1 } \\
\text { III:0 } \\
\text { IV:1 } \\
\text { Total: } 3\end{array}$ & USA & $\begin{array}{l}\text { Examined American } \\
\text { Indian college students' } \\
\text { suicidality. } \\
\text { Cross-sectional survey. }\end{array}$ & 272 & not reported & not reported & $\begin{array}{l}\text { Suicidal Risk Questionnaire } \\
\text { (SRQ), Cultural commitment } \\
\text { and demographic } \\
\text { questionnaire, Suicide-related } \\
\text { beliefs and help-seeking } \\
\text { preferences questionnaire. }\end{array}$ & $\begin{array}{l}\text { Suicidal ideation comparable } \\
\text { with general college students. } \\
\text { Low awareness of traditional } \\
\text { tribal suicide }(10 \%) \text {. } \\
\text { Participants more committed } \\
\text { to tribal culture prefer } \\
\text { counselling from American } \\
\text { Indian persons. }\end{array}$ \\
\hline $\begin{array}{l}\text { Wong et al., } \\
2012 \text { [42] }\end{array}$ & $\begin{array}{l}\text { I:3 } \\
\text { II:1 } \\
\text { III:0 } \\
\text { IV:2 } \\
\text { Total: } 6\end{array}$ & USA & $\begin{array}{l}\text { Investigated youth risk } \\
\text { factors for suicide in } \\
\text { ethnic minorities } \\
\text { among American high } \\
\text { school students. } \\
\text { Cross-sectional survey }\end{array}$ & 88,532 & not reported & not reported & $\begin{array}{l}\text { Data from the 1999-2009 Youth } \\
\text { Risk Behaviour Surveys } \\
\text { (YRBS), a national survey of } \\
\text { high school students. } \\
\text { Questionnaire. }\end{array}$ & $\begin{array}{l}\text { Native Hawaiian/Pacific } \\
\text { Islander adolescents and } \\
\text { multiracial adolescents have a } \\
\text { higher prevalence of risk } \\
\text { factors for suicide. }\end{array}$ \\
\hline $\begin{array}{l}\text { Hunt et al., } 2003 \\
\text { [43] }\end{array}$ & $\begin{array}{l}\text { I:1 } \\
\text { II:1 } \\
\text { III:1 } \\
\text { IV:2 } \\
\text { Total: } 5\end{array}$ & UK & $\begin{array}{l}\text { Investigated suicides in } \\
\text { ethnic minorities within } \\
12 \text { months of contact } \\
\text { with mental health } \\
\text { services in England and } \\
\text { Wales. Clinical survey }\end{array}$ & 282 & not reported & 282 & $\begin{array}{l}\text { Data from the Office for } \\
\text { National Statistics (ONS) and } \\
\text { questionnaire. }\end{array}$ & $\begin{array}{l}\text { Among patients from ethnic } \\
\text { minorities who had been in } \\
\text { contact with mental health } \\
\text { services in the } 12 \text { months } \\
\text { before death, suicide was } \\
\text { characterised by: violent } \\
\text { methods, first episode of } \\
\text { self-harm, high rates of } \\
\text { schizophrenia, unemployment, } \\
\text { history of violence, and drug } \\
\text { misuse }\end{array}$ \\
\hline $\begin{array}{l}\text { Bhui et al., } 2012 \\
\text { [44] }\end{array}$ & $\begin{array}{l}\text { I:2 } \\
\text { II:1 } \\
\text { III:1 } \\
\text { IV:2 } \\
\text { Total: } 6\end{array}$ & UK & $\begin{array}{l}\text { Investigated the } \\
\text { influence of ethnicity } \\
\text { on suicide, and related } \\
\text { risk indicators among } \\
\text { suicidal patients in } \\
\text { contact with psychiatric } \\
\text { services. Retrospective. }\end{array}$ & 1358 & not reported & 1358 & $\begin{array}{l}\text { Data from the United } \\
\text { Kingdom's Office for National } \\
\text { Statistics between } 1996 \text { and } \\
\text { 2001. Questionnaire. }\end{array}$ & $\begin{array}{l}\text { Black African men have higher } \\
\text { rates of suicide as compared } \\
\text { with the white British group. } \\
\text { Classical indicators of suicide } \\
\text { risk are less common in black } \\
\text { Africans and South Asians, as } \\
\text { compared with the white } \\
\text { British group. }\end{array}$ \\
\hline
\end{tabular}


Table 1. Cont.

\begin{tabular}{|c|c|c|c|c|c|c|c|c|}
\hline $\begin{array}{l}\text { Bhui et al., } 2008 \\
\text { [39] }\end{array}$ & $\begin{array}{l}\text { Quality Score } \\
\text { I:2 } \\
\text { II:1 } \\
\text { III:1 } \\
\text { IV:2 } \\
\text { Total: } 6\end{array}$ & Country & $\begin{array}{l}\text { Aim and Study Design } \\
\text { Investigated suicide } \\
\text { rates, symptoms, and } \\
\text { preventability of } \\
\text { suicide among suicidal } \\
\text { patients within } 12 \\
\text { months of contact with } \\
\text { mental health services. } \\
\text { Retrospective. }\end{array}$ & Sample & not reported & Suicide $N$ & $\begin{array}{l}\text { Methods } \\
\text { Data from the United } \\
\text { Kingdom's Office for National } \\
\text { Statistics. }\end{array}$ & $\begin{array}{l}\text { Rates and SMRs varied across } \\
\text { ethnic groups. }\end{array}$ \\
\hline $\begin{array}{l}\text { Ngwena, } 2014 \\
\text { [45] }\end{array}$ & $\begin{array}{l}\text { I:1 } \\
\text { II:1 } \\
\text { III:1 } \\
\text { IV:2 } \\
\text { Total: } 5\end{array}$ & UK & $\begin{array}{l}\text { Investigated trends of } \\
\text { suicides in black and } \\
\text { minority ethnic (BME) } \\
\text { groups. Retrospective. }\end{array}$ & 192 & not reported & 192 & $\begin{array}{l}\text { Data from the Office of } \\
\text { National Statistics (ONS) and } \\
\text { Public Health Observatory } \\
\text { Mortality files, over the periods } \\
2009-2012 \text { and 2010-2013. }\end{array}$ & $\begin{array}{l}\text { Suicides among patients from } \\
\text { black and minority ethnic } \\
\text { (BME) groups most prevalent } \\
\text { in those of Arab origin and } \\
\text { North or South Americans } \\
(28 \%) \text {, followed by those of } \\
\text { Western and Eastern European } \\
\text { origin }(26 \%) .\end{array}$ \\
\hline $\begin{array}{l}\text { Walker et al., } \\
2005 \text { [46] }\end{array}$ & $\begin{array}{l}\text { I:1 } \\
\text { II:1 } \\
\text { III:0 } \\
\text { IV:1 } \\
\text { Total: } 3\end{array}$ & USA & $\begin{array}{l}\text { Investigated the role of } \\
\text { acculturation in } \\
\text { suicidal-behaviour } \\
\text { among African } \\
\text { descendants living in } \\
\text { the USA. } \\
\text { Cross-sectional survey }\end{array}$ & 423 & - & not reported & $\begin{array}{l}\text { African American } \\
\text { Acculturation Scale (AAAS). } \\
\text { The Multi-Dimensional } \\
\text { Support Scale (MDSS). The } \\
\text { Spiritual Well-Being Scale } \\
\text { (SWBS) }\end{array}$ & $\begin{array}{l}\text { Religious well being, and not } \\
\text { acculturation, is predictive of } \\
\text { suicidal ideation and history of } \\
\text { suicide attempt. }\end{array}$ \\
\hline \multicolumn{9}{|l|}{ Immigrants } \\
\hline \multicolumn{9}{|l|}{$\begin{array}{l}\text { Continent of } \\
\text { Origin: Asia }\end{array}$} \\
\hline $\begin{array}{l}\text { Chung et al., } \\
2015 \text { [47] }\end{array}$ & $\begin{array}{l}\text { I: } 1 \\
\text { II:1 } \\
\text { III:1 } \\
\text { IV:1 } \\
\text { Total: } 4\end{array}$ & USA & $\begin{array}{l}\text { Risks and protective } \\
\text { factors among Asian } \\
\text { immigrants who } \\
\text { repeatedly attempt } \\
\text { suicide. Mixed-method } \\
\text { study: Study } 1= \\
\text { retrospective study and } \\
\text { Study } 2 \text { = clinical } \\
\text { survey. }\end{array}$ & $\begin{array}{l}\text { Study } 1=44 \\
\text { Study } 2=12\end{array}$ & $\geq 2-5 /$ persons & 0 & $\begin{array}{l}\text { Retrospective study ( } n=44: \\
\text { clinical records) and clinical } \\
\text { survey ( } n=12 \text { semi-structured } \\
\text { interviews). }\end{array}$ & $\begin{array}{l}\text { Among Asian immigrants with } \\
\text { repeated suicide attempts, risk } \\
\text { factors are: hopelessness, social } \\
\text { isolation, self-stigma, feelings } \\
\text { of failure, and sense of } \\
\text { rejection by own family. } \\
\text { Protective factors: } \\
\text { psychological well-being, } \\
\text { feeling cared for and able to } \\
\text { reciprocate care for others. }\end{array}$ \\
\hline $\begin{array}{l}\text { Bhugra et al., } \\
2002 \text { [36] }\end{array}$ & $\begin{array}{l}\text { I:1 } \\
\text { II:1 } \\
\text { III:1 } \\
\text { IV:2 } \\
\text { Total: } 5\end{array}$ & UK & $\begin{array}{l}\text { Collected information } \\
\text { on inception rates of } \\
\text { attempted suicides } \\
\text { across all ethnic groups, } \\
\text { in the UK. } \\
\text { Cross-sectional survey. }\end{array}$ & 434 & 65 & 0 & $\begin{array}{l}\text { Data from the } 1991 \text { census. } \\
\text { Semi-structured interview } \\
\text { (schedule assessing the } \\
\text { attempt, culture identity } \\
\text { schedule, life events, GHQ-28, } \\
\text { and Clinical Interview } \\
\text { Schedule-R). }\end{array}$ & $\begin{array}{l}\text { South Asian women, especially } \\
\text { those aged } 18-24 \text {, have higher } \\
\text { rates of attempted suicide, in } \\
\text { association with high rates of } \\
\text { cultural alienation and } \\
\text { previous attempts. }\end{array}$ \\
\hline
\end{tabular}


Table 1. Cont.

\begin{tabular}{|c|c|c|c|c|c|c|c|c|}
\hline Study & Quality Score & Country & Aim and Study Design & Sample & Attempt $N$ & Suicide $N$ & Methods & Main Findings \\
\hline $\begin{array}{l}\text { Dai et al., } 2015 \\
\text { [21] }\end{array}$ & $\begin{array}{l}\text { I:2 } \\
\text { II:1 } \\
\text { III:1 } \\
\text { IV:1 } \\
\text { Total: } 5\end{array}$ & China & $\begin{array}{l}\text { Explored psychological } \\
\text { consequences of } \\
\text { internal migration } \\
\text { among young rural } \\
\text { Chinese and the } \\
\text { associations between } \\
\text { migrant status, mental } \\
\text { health, and suicidal } \\
\text { behaviours. } \\
\text { Cross-sectional survey. }\end{array}$ & 1646 & 10 & 0 & $\begin{array}{l}\text { Structured interview. } \\
\text { Questionnaire (psycho-QOL } \\
\text { subscale of the World Health } \\
\text { Organization's QOL } \\
\text { Questionnaire-Brief Version; } \\
\text { CEDS). }\end{array}$ & $\begin{array}{l}\text { Socio-demographic and clinical } \\
\text { variables, and social support, } \\
\text { not migrant status, were the } \\
\text { central determinants of mental } \\
\text { health among all participants. } \\
\text { Compared to their } \\
\text { rural-residing peers, migrant } \\
\text { workers had a decreased risk } \\
\text { for depression and comparable } \\
\text { risk for poor psycho-QOL and } \\
\text { one-year serious suicide } \\
\text { ideation. }\end{array}$ \\
\hline $\begin{array}{l}\text { Ayalon, } 2012 \\
\text { [33] }\end{array}$ & $\begin{array}{l}\text { I:1 } \\
\text { II:1 } \\
\text { III:1 } \\
\text { IV:1 } \\
\text { Total: } 4\end{array}$ & Israel & $\begin{array}{l}\text { Investigated suicidal } \\
\text { and depressive } \\
\text { symptoms along with } \\
\text { exposure to abuse and } \\
\text { perceived social } \\
\text { support in Filipino } \\
\text { home care workers in } \\
\text { Israel. Cross-sectional } \\
\text { survey. }\end{array}$ & 178 & 8 & - & $\begin{array}{l}\text { Questionnaires (Paykel Suicide } \\
\text { Scale; Patient Health } \\
\text { Questionnaire-9). }\end{array}$ & $\begin{array}{l}\text { The Filipino sample in Israel } \\
\text { showed higher levels of suicide } \\
\text { attempts compared to national } \\
\text { statistics in the Philippines. } \\
\text { Abuse within the home/work } \\
\text { environment ( } 35 \% \text { of the } \\
\text { sample) was predictive for } \\
\text { depressive symptoms (3.4\% } \\
\text { depressed) }\end{array}$ \\
\hline $\begin{array}{l}\text { Hagaman et al., } \\
2016 \text { [48] }\end{array}$ & $\begin{array}{l}\text { I:1 } \\
\text { II:1 } \\
\text { III:1 } \\
\text { IV:1 } \\
\text { Total: } 4\end{array}$ & USA & $\begin{array}{l}\text { Explored suicides } \\
\text { among Bhutanese } \\
\text { refugees in the USA. } \\
\text { Cross-sectional survey. }\end{array}$ & 14 & not reported & 14 & $\begin{array}{l}\text { Psychological autopsies, } \\
\text { Hopkins Symptom } \\
\text { Checklist-25 (HSC). }\end{array}$ & $\begin{array}{l}\text { Suicide among Bhutanese } \\
\text { refugees is connected with } \\
\text { experiences of family } \\
\text { withdrawal, integration } \\
\text { difficulties, and perceived lack } \\
\text { of care. }\end{array}$ \\
\hline $\begin{array}{l}\text { Duldulao et al., } \\
2009 \text { [35] }\end{array}$ & $\begin{array}{l}\text { I:2 } \\
\text { II:2 } \\
\text { III:1 } \\
\text { IV:2 } \\
\text { Total: } 7\end{array}$ & USA & $\begin{array}{l}\text { Investigated suicidal } \\
\text { behaviours among } \\
\text { Asian Americans, } \\
\text { focusing on the } \\
\text { correlates of suicidal } \\
\text { ideation, plan, and } \\
\text { attempt with nativity } \\
\text { and gender. } \\
\text { Prospective study. }\end{array}$ & 2095 & 52 & - & $\begin{array}{l}\text { Interviews. Data from the } \\
\text { National Latino and Asian } \\
\text { American Study (NLAAS). }\end{array}$ & $\begin{array}{l}\text { U.S.-born Asian American } \\
\text { women showed higher } \\
\text { prevalence of suicidal ideation } \\
\text { and suicide plan than } \\
\text { U.S.-born Asian American men } \\
\text { and immigrant Asian } \\
\text { American men and women. }\end{array}$ \\
\hline $\begin{array}{l}\text { Wong et al., } \\
2014 \text { [37] }\end{array}$ & $\begin{array}{l}\text { I:2 } \\
\text { II:1 } \\
\text { III:0 } \\
\text { IV:2 } \\
\text { Total: } 5\end{array}$ & USA & $\begin{array}{l}\text { Explored the } \\
\text { correlation between the } \\
\text { proportion of life in the } \\
\text { USA and suicide } \\
\text { ideation among Asian } \\
\text { Americans in order to } \\
\text { address within-group } \\
\text { ethnic variability. } \\
\text { Cross-sectional survey. }\end{array}$ & 1332 & - & & $\begin{array}{l}\text { Data from the National } \\
\text { Epidemiologic Survey of } \\
\text { Alcohol and Related } \\
\text { Conditions. }\end{array}$ & $\begin{array}{l}\text { Asian Americans in the U.S. } \\
\text { had higher rate of suicide } \\
\text { ideation, with significant } \\
\text { differences within ethnic } \\
\text { groups: the highest rates were } \\
\text { found in Korean Americans } \\
\text { and the lowest in Indian } \\
\text { Americans. }\end{array}$ \\
\hline
\end{tabular}


Table 1. Cont.

\begin{tabular}{|c|c|c|c|c|c|c|c|c|}
\hline Study & Quality Score & Country & Aim and Study Design & Sample & Attempt $N$ & Suicide $N$ & Methods & Main Findings \\
\hline \multicolumn{9}{|l|}{$\begin{array}{l}\text { Continent of } \\
\text { Origin: The } \\
\text { Americas }\end{array}$} \\
\hline Eaton, 2011 [49] & $\begin{array}{l}\text { I:2 } \\
\text { II: } 1 \\
\text { III: } 1 \\
\text { IV: } 2 \\
\text { Total: } 6\end{array}$ & USA & $\begin{array}{l}\text { Investigated the } \\
\text { associations between } \\
\text { racial/ethnic variations } \\
\text { and risk of suicidal } \\
\text { behaviours among } \\
\text { Hispanic/Latina female } \\
\text { students. } \\
\text { Cross-sectional survey. }\end{array}$ & 6322 & $\begin{array}{l}\text { Total: } 575 \\
\text { White } 318 \\
\text { African } \\
\text { american } 101 \\
\text { Hispanic } 161\end{array}$ & - & $\begin{array}{l}\text { Data from The } 2007 \text { national } \\
\text { school-based Youth Risk } \\
\text { Behaviour Survey (YRBS); }\end{array}$ & $\begin{array}{l}\text { Hispanic/Latina female } \\
\text { students had a higher } \\
\text { prevalence of suicidal ideation } \\
(21.1 \%) \text { and suicide attempts } \\
(14.0 \%) \text { than white and African } \\
\text { American students. The risk } \\
\text { behaviours associated with } \\
\text { suicidal ideation and suicide } \\
\text { attempts were: injuries and } \\
\text { violence; tobacco use; alcohol } \\
\text { and drug use; sexual } \\
\text { behaviours; perceived health } \\
\text { status. }\end{array}$ \\
\hline $\begin{array}{l}\text { Peña et al., } 2008 \\
\text { [50] }\end{array}$ & $\begin{array}{l}\text { I:2 } \\
\text { II:2 } \\
\text { III:1 } \\
\text { IV: } 2 \\
\text { Total: } 7\end{array}$ & USA & $\begin{array}{l}\text { Investigated the } \\
\text { associations between } \\
\text { immigration generation } \\
\text { status and suicide } \\
\text { attempts, substance use } \\
\text { and depressive } \\
\text { symptoms among } \\
\text { Latino adolescents in } \\
\text { the USA. } \\
\text { Cross-sectional survey } \\
\text { (prospective cohort } \\
\text { study). }\end{array}$ & 3135 & $\begin{array}{l}\text { I generation } \\
53 \\
\text { II generation } \\
106 \\
\text { Later } \\
\text { generation } 153\end{array}$ & - & $\begin{array}{l}\text { Data from The National } \\
\text { Longitudinal Study of } \\
\text { Adolescent Health (Add } \\
\text { Health). Interviews. }\end{array}$ & $\begin{array}{l}\text { Among Latino adolescents, } \\
\text { immigrant generation status is } \\
\text { determinant for suicide } \\
\text { attempts. Second-generation } \\
\text { U.S.-born Latino youths were } \\
2.87 \text { times more likely to have } \\
\text { made suicide attempts as } \\
\text { compared to foreign-born } \\
\text { youth. Later generations of } \\
\text { U.S.-born Latinos were } 3.57 \\
\text { times more likely to have made } \\
\text { suicide attempts as compared } \\
\text { to first-generation Latino } \\
\text { youths. }\end{array}$ \\
\hline $\begin{array}{l}\text { Hovey et al., } \\
2003 \text { [51] }\end{array}$ & $\begin{array}{l}\text { I: } 1 \\
\text { II: } 2 \\
\text { III:0 } \\
\text { IV: } 1 \\
\text { Total: } 4\end{array}$ & USA & $\begin{array}{l}\text { Investigated suicide } \\
\text { risk factors and } \\
\text { depressive symptoms } \\
\text { among Mexican } \\
\text { migrant women } \\
\text { farmworkers in the U.S. } \\
\text { Midwest. Prospective } \\
\text { study. }\end{array}$ & 20 & - & - & $\begin{array}{l}\text { Interview and questionnaires } \\
\text { (Adult Self- Perception Scale, } \\
\text { Family Assessment Device, } \\
\text { Beck Hopelessness Scale, SAFE } \\
\text { Scale, The Personal Resource } \\
\text { Questionnaire, Personality } \\
\text { Assessment Inventory, Suicidal } \\
\text { Ideation and Interview Topics, } \\
\text { Center for Epidemiologic } \\
\text { Studies Depression Scale). }\end{array}$ & $\begin{array}{l}\text { Migrant women farmworkers } \\
\text { presented elevated levels of } \\
\text { anxiety, depression, and } \\
\text { suicidal ideation. High } \\
\text { depression symptoms were } \\
\text { associated with: family } \\
\text { dysfunction, ejective social } \\
\text { support, hopelessness, and } \\
\text { acculturative stress. High } \\
\text { suicidal ideation was } \\
\text { associated with lower } \\
\text { self-esteem, family } \\
\text { dysfunction, less ejective social } \\
\text { support, hopelessness, } \\
\text { acculturative stress. }\end{array}$ \\
\hline
\end{tabular}


Table 1. Cont.

\begin{tabular}{|c|c|c|c|c|c|c|c|c|}
\hline Study & Quality Score & Country & Aim and Study Design & Sample & Attempt $N$ & Suicide $N$ & Methods & Main Findings \\
\hline $\begin{array}{l}\text { Borges et al., } \\
2009[52]\end{array}$ & $\begin{array}{l}\text { I:2 } \\
\text { II:1 } \\
\text { III:1 } \\
\text { IV: } 2 \\
\text { Total: } 6\end{array}$ & USA & $\begin{array}{l}\text { Explored suicidal } \\
\text { behaviour among } \\
\text { Mexicans and Mexican } \\
\text { Americans. } \\
\text { Retrospective study. }\end{array}$ & $\begin{array}{l}n 1=5782 ; n 2 \\
=1284\end{array}$ & - & 1284 & $\begin{array}{l}\text { Data from the Mexican } \\
\text { National Comorbidity Survey } \\
\text { (MNCS) (2001-2002; } n 1=5782) \\
\text { and the Collaborative } \\
\text { Psychiatric Epidemiology } \\
\text { Surveys (CPES): (2001-2003; } n 2 \\
=1284) .\end{array}$ & $\begin{array}{l}\text { Migration to the USA is a risk } \\
\text { factor for suicidal behaviour } \\
\text { among Mexican people. Risk } \\
\text { for suicidal ideation: having a } \\
\text { family member in the USA; } \\
\text { having arrived in the USA } \\
\text { before the age of 12, and being } \\
\text { U.S.-born. Risk for suicide } \\
\text { attempts: having a family } \\
\text { member in the USA and being } \\
\text { U.S.-born. }\end{array}$ \\
\hline $\begin{array}{l}\text { Sorenson et al., } \\
1999 \text { [53] }\end{array}$ & $\begin{array}{l}\text { I:3 } \\
\text { II:1 } \\
\text { III:1 } \\
\text { IV: } 2 \\
\text { Total: } 7\end{array}$ & USA & Cross-sectional survey & 38,166 & - & 1344 & $\begin{array}{l}\text { Data from California Master } \\
\text { Mortality. }\end{array}$ & $\begin{array}{l}\text { The differences in mortality } \\
\text { between foreign- and U.S.-born } \\
\text { persons: young immigrants } \\
\text { have lower or similar risk of } \\
\text { death and they are } \\
\text { underrepresented in suicides } \\
\text { and overrepresented in } \\
\text { homicides compared with } \\
\text { U.S.-born persons. }\end{array}$ \\
\hline \multicolumn{9}{|l|}{$\begin{array}{l}\text { Continent of } \\
\text { Origin: Africa }\end{array}$} \\
\hline $\begin{array}{l}\text { Shoval et al., } \\
2007 \text { [22] }\end{array}$ & $\begin{array}{l}\text { I:0 } \\
\text { II:1 } \\
\text { III:1 } \\
\text { IV:2 } \\
\text { Total: } 4\end{array}$ & Israel & $\begin{array}{l}\text { Investigated suicide in } \\
\text { Ethiopian immigrants. }\end{array}$ & - & - & $25 / 100,000$ & $\begin{array}{l}\text { Data from National } \\
\text { epidemiological surveys by the } \\
\text { Israeli Ministry of Health. }\end{array}$ & $\begin{array}{l}\text { High rates of suicide among } \\
\text { Ethiopian immigrants in Israel, } \\
\text { significantly higher than other } \\
\text { immigrant populations. }\end{array}$ \\
\hline $\begin{array}{l}\text { Walsh et al., } \\
2012 \text { [54] }\end{array}$ & $\begin{array}{l}\text { I:1 } \\
\text { II:1 } \\
\text { III:0 } \\
\text { IV:1 } \\
\text { Total: } 3\end{array}$ & Israel & $\begin{array}{l}\text { Investigated the } \\
\text { relations between } \\
\text { suicidal ideation and } \\
\text { alcohol abuse with } \\
\text { ethnic identity and } \\
\text { parental support } \\
\text { among Ethiopian } \\
\text { adolescents in Israel. } \\
\text { Cross-sectional survey. }\end{array}$ & 200 & - & - & Questionnaires. & $\begin{array}{l}\text { Suicidal ideation correlates } \\
\text { with ethnic identity, alcohol } \\
\text { use and parental support. A } \\
\text { strong positive ethnic identity } \\
\text { plays a protective role against } \\
\text { suicidal and risk behaviours. } \\
\text { Among Ethiopian adolescents } \\
\text { in Israel, Ethiopian identity } \\
\text { correlates with lower levels of } \\
\text { suicidal ideation and alcohol } \\
\text { use. }\end{array}$ \\
\hline $\begin{array}{l}\text { ntinent of } \\
\text { rigin: Europe }\end{array}$ & & & & & & & & \\
\hline
\end{tabular}


Table 1. Cont

\begin{tabular}{|c|c|c|c|c|c|c|c|c|}
\hline Study & Quality Score & Country & Aim and Study Design & Sample & Attempt $N$ & Suicide $N$ & Methods & Main Findings \\
\hline $\begin{array}{l}\text { Aichberger et } \\
\text { al., } 2015 \text { [55] }\end{array}$ & $\begin{array}{l}\text { I: } 1 \\
\text { II:1 } \\
\text { III: } 1 \\
\text { IV:1 } \\
\text { Total: } 4\end{array}$ & Germany & $\begin{array}{l}\text { Investigated rates, } \\
\text { motives and } \\
\text { effectiveness of } \\
\text { intervention-programs } \\
\text { for suicidal behaviour } \\
\text { among Turkish women } \\
\text { in Berlin who made } \\
\text { suicide attempts. } \\
\text { Cross-sectional survey. }\end{array}$ & 159 & 159 & - & Questionnaires. & $\begin{array}{l}\text { The high rate of suicide } \\
\text { attempts among } \\
\text { second-generation Turkish } \\
\text { women in Berlin showed a } \\
\text { significant reduction due to the } \\
\text { application of the intervention } \\
\text { program. }\end{array}$ \\
\hline $\begin{array}{l}\text { Ryan et al., } 2006 \\
\text { [31] }\end{array}$ & $\begin{array}{l}\text { I:1 } \\
\text { II:2 } \\
\text { III:0 } \\
\text { IV:1 } \\
\text { Total: } 4\end{array}$ & UK & $\begin{array}{l}\text { Explored depression } \\
\text { and suicide rates in } \\
\text { Irish migrants in } \\
\text { London. Case-control } \\
\text { study }\end{array}$ & 360 & - & - & $\begin{array}{l}\text { Questionnaire (Beck } \\
\text { Depression Inventory (BDI). }\end{array}$ & $\begin{array}{l}\text { Irish migrants in London show } \\
\text { higher rates of depression and } \\
\text { suicide compared with other } \\
\text { minority ethnic groups, } \\
\text { especially when associated } \\
\text { with poorly planned migration. } \\
\text { This effect can be modified by } \\
\text { positive post-migration } \\
\text { experiences. }\end{array}$ \\
\hline $\begin{array}{l}\text { Shah et al. A, } \\
2011 \text { [38] }\end{array}$ & $\begin{array}{l}\text { I:0 } \\
\text { II:1 } \\
\text { III:0 } \\
\text { IV:2 } \\
\text { Total: } 3\end{array}$ & UK & $\begin{array}{l}\text { Examined suicide rates } \\
\text { in England and Wales } \\
\text { and its correlation with } \\
\text { ethnicity. Retrospective. }\end{array}$ & - & - & - & $\begin{array}{l}\text { Data from the Office of } \\
\text { National Statistics. }\end{array}$ & $\begin{array}{l}\text { Differences in suicide rates } \\
\text { among ethnic groups: male } \\
\text { suicide rates were higher in all } \\
\text { ethnic groups, except in the } \\
\text { Chinese group, and increased } \\
\text { with ageing among Indians; } \\
\text { female suicide rates were } \\
\text { higher among Chinese and } \\
\text { increased with ageing among } \\
\text { the African and Chinese } \\
\text { groups. }\end{array}$ \\
\hline $\begin{array}{l}\text { Voracek et al., } \\
2009[56]\end{array}$ & $\begin{array}{l}\text { I:3 } \\
\text { II:1 } \\
\text { III:1 } \\
\text { IV:2 } \\
\text { Total: } 7\end{array}$ & Austria & $\begin{array}{l}\text { Investigated the role of } \\
\text { genetic risk factors for } \\
\text { suicide, comparing } \\
\text { immigrant suicide rates } \\
\text { in Austria with } \\
\text { country-of-birth ones. } \\
\text { Retrospective. }\end{array}$ & 65,206 & - & 65,206 & Data from Statistics Austria. & $\begin{array}{l}\text { Immigrant and homeland } \\
\text { suicide rates were significantly } \\
\text { positively associated. This } \\
\text { evidence confirms the } \\
\text { existence of genetic risk factors } \\
\text { for suicide specific for each } \\
\text { population. }\end{array}$ \\
\hline $\begin{array}{l}\text { Termorshuizen } \\
\text { et al., } 2012 \text { [24] }\end{array}$ & $\begin{array}{l}\text { I: } 3 \\
\text { II:1 } \\
\text { III:1 } \\
\text { IV:2 } \\
\text { Total: } 7\end{array}$ & Netherlands & $\begin{array}{l}\text { Examined ethnic } \\
\text { density and suicide risk } \\
\text { among migrant groups } \\
\text { in four big cities in the } \\
\text { Netherlands. } \\
\text { Retrospective cohort } \\
\text { study. }\end{array}$ & $2,874,464$ & - & 2572 & $\begin{array}{l}\text { Data from Statistics } \\
\text { Netherlands. }\end{array}$ & $\begin{array}{l}\text { The ethnic density influenced } \\
\text { suicide risk among ethnic } \\
\text { groups. The presence of their } \\
\text { own ethnic group in the } \\
\text { neighbourhood has a positive } \\
\text { effect on suicide risk among } \\
\text { non-Western minorities. }\end{array}$ \\
\hline
\end{tabular}


Table 1. Cont.

\begin{tabular}{|c|c|c|c|c|c|c|c|c|}
\hline $\begin{array}{l}\text { Study } \\
\text { Di Thiene et al., } \\
2015 \text { [25] }\end{array}$ & $\begin{array}{l}\text { Quality Score } \\
\text { I:3 } \\
\text { II:2 } \\
\text { III: } 1 \\
\text { IV:2 } \\
\text { Total: } 8\end{array}$ & Country & $\begin{array}{l}\text { Aim and Study Design } \\
\text { Investigated differences } \\
\text { in suicide between first- } \\
\text { and second-generation } \\
\text { immigrants in Sweden. } \\
\text { Prospective } \\
\text { population-based } \\
\text { cohort study. }\end{array}$ & $4,034,728$ & Attempt $N$ & Suicide $N$ & $\begin{array}{l}\text { Methods } \\
\text { Data from Statistics Swede: } \\
\text { theNational Board of Health } \\
\text { and Welfare. }\end{array}$ & $\begin{array}{l}\text { Main Findings } \\
\text { The risk of suicide is lower in } \\
\text { the first generation and higher } \\
\text { in the second generation of } \\
\text { immigrants compared with } \\
\text { natives in Sweden. }\end{array}$ \\
\hline $\begin{array}{l}\text { Johansonn, } 1997 \\
\text { [57] }\end{array}$ & $\begin{array}{l}\text { I: } 3 \\
\text { II:1 } \\
\text { III:0 } \\
\text { IV:2 } \\
\text { Total: } 6\end{array}$ & Sweden & $\begin{array}{l}\text { Analysed the influence } \\
\text { of ethnicity, age, sex, } \\
\text { marital status, and date } \\
\text { of immigration on } \\
\text { suicide rates among } \\
\text { immigrants in Sweden. } \\
\text { Retrospective. }\end{array}$ & $6,725,274$ & - & - & $\begin{array}{l}\text { Data from Central Cause of } \\
\text { Death Register. }\end{array}$ & $\begin{array}{l}\text { Risk factors for suicide among } \\
\text { immigrants in Sweden: } \\
\text { ethnicity, being unmarried, } \\
\text { male sex, age } 45-54 \text { or } 75 \text { and } \\
\text { older, being born in Eastern } \\
\text { Europe, Finland, or in } \\
\text { non-European countries, and } \\
\text { having immigrated to Sweden } \\
\text { in } 1967 \text { or earlier. Males born } \\
\text { in Russia/Finland and females } \\
\text { born in Hungary/ Russia/ } \\
\text { Finland/ Poland showed the } \\
\text { highest risk ratios for suicide } \\
\text { and higher risks than in their } \\
\text { countries of birth. }\end{array}$ \\
\hline Stack, 1981 [58] & $\begin{array}{l}\text { I:0 } \\
\text { II:2 } \\
\text { III:0 } \\
\text { IV:2 } \\
\text { Total: } 4\end{array}$ & USA & $\begin{array}{l}\text { Examined the } \\
\text { association between } \\
\text { immigration and } \\
\text { suicide. Systematic } \\
\text { cross-national } \\
\text { investigation. }\end{array}$ & - & - & - & Data from the World Bank. & $\begin{array}{l}\text { The rate of immigration has } \\
\text { effects on the incidence of } \\
\text { suicide: } 1 \% \text { increase in } \\
\text { immigration is associated with } \\
\text { a } 13 \% \text { increase in the rate of } \\
\text { suicide. }\end{array}$ \\
\hline $\begin{array}{l}\text { Burvill, } 1998 \\
\text { [59] }\end{array}$ & $\begin{array}{l}\text { I:3 } \\
\text { II:2 } \\
\text { III:0 } \\
\text { IV:1 } \\
\text { Total: } 6\end{array}$ & Australia & $\begin{array}{l}\text { Investigate suicide rates } \\
\text { in migrant groups to } \\
\text { Australia from Britain, } \\
\text { Ireland, and the rest of } \\
\text { Europe for the years } \\
\text { 1979-1990. } \\
\text { Epidemiological study. }\end{array}$ & - & - & - & $\begin{array}{l}\text { Data from the Australian } \\
\text { Bureau of statics (ABS) and the } \\
\text { World Health Organization } \\
\text { Annual Statistics between } 1979 \\
\text { and } 1990 .\end{array}$ & $\begin{array}{l}\text { Comparison between the } \\
\text { Australian-born and migrants } \\
\text { from } 11 \text { European countries } \\
\text { showed increased rates in } \\
\text { immigrants compared with } \\
\text { those in their country of birth } \\
\text { (COB), and a significant rank } \\
\text { correlation of the immigrant } \\
\text { rates with those in their COB. }\end{array}$ \\
\hline $\begin{array}{l}\text { Värnik et al., } \\
2005[60]\end{array}$ & $\begin{array}{l}\text { I:3 } \\
\text { II:2 } \\
\text { III:0 } \\
\text { IV:1 } \\
\text { Total: } 6\end{array}$ & Estonia & $\begin{array}{l}\text { Investigated changes in } \\
\text { suicide rates among } \\
\text { Russians in Estonia } \\
\text { before (1983-1990) and } \\
\text { after (1991-1998) } \\
\text { Estonian independence } \\
\text { from the Soviet Union. } \\
\text { Epidemiological study. }\end{array}$ & - & - & - & $\begin{array}{l}\text { Data from the World Health } \\
\text { Organization and the Estonian } \\
\text { Statistical Office. }\end{array}$ & $\begin{array}{l}\text { Significantly higher suicide } \\
\text { rate in Estonian Russians after } \\
\text { Estonian independence in 1991, } \\
\text { as compared to that of } \\
\text { Estonians and Russians in } \\
\text { Russia. The loss of privileged } \\
\text { status of Russians during the } \\
\text { Soviet era led to increasing } \\
\text { suicide rates. }\end{array}$ \\
\hline
\end{tabular}


Table 1. Cont.

\begin{tabular}{|c|c|c|c|c|c|c|c|c|}
\hline Study & Quality Score & Country & Aim and Study Design & Sample & Attempt $N$ & Suicide $N$ & Methods & Main Findings \\
\hline $\begin{array}{l}\text { Maynard et al., } \\
2012 \text { [61] }\end{array}$ & $\begin{array}{l}\text { I:0 } \\
\text { II:1 } \\
\text { III:0 } \\
\text { IV:2 } \\
\text { Total: } 3\end{array}$ & UK & $\begin{array}{l}\text { Analysed suicide in } \\
\text { England and Wales } \\
\text { among immigrants. } \\
\text { Cross-sectional survey. }\end{array}$ & & & 0 & $\begin{array}{l}\text { Data from The Office for } \\
\text { National Statistics (ONS). }\end{array}$ & $\begin{array}{l}\text { This study indicated declining } \\
\text { trends of suicide rates for most } \\
\text { migrant groups and for } \\
\text { English and Welsh-born } \\
\text { women, but adverse trends for } \\
\text { some country of birth groups. }\end{array}$ \\
\hline \multicolumn{9}{|l|}{$\begin{array}{l}\text { Continent of } \\
\text { Origin: } \\
\text { Australia }\end{array}$} \\
\hline $\begin{array}{l}\text { Taylor et al., } \\
1998 \text { [62] }\end{array}$ & $\begin{array}{l}\text { I:0 } \\
\text { II:1 } \\
\text { III:0 } \\
\text { IV:2 } \\
\text { Total: } 3\end{array}$ & Australia & $\begin{array}{l}\text { Examined the } \\
\text { differences in suicide } \\
\text { by socio-economic } \\
\text { status (SES) in urban } \\
\text { areas of Australia. } \\
\text { Retrospective. }\end{array}$ & - & - & - & $\begin{array}{l}\text { Data from the NSW unit record } \\
\text { mortality tape, from the } \\
\text { Australian Bureau of Statistics } \\
\text { (ABS). }\end{array}$ & $\begin{array}{l}\text { Risk of suicide in females was } \\
28 \% \text { that of males in adults, } \\
\text { and } 21 \% \text { for youth. The risk } \\
\text { increased significantly with } \\
\text { decreasing socio-economic } \\
\text { status in males, but not in } \\
\text { females and depended on the } \\
\text { country of origin. }\end{array}$ \\
\hline $\begin{array}{l}\text { Morrell et al., } \\
1999 \text { [63] }\end{array}$ & $\begin{array}{l}\text { I:0 } \\
\text { II:2 } \\
\text { III:0 } \\
\text { IV:2 } \\
\text { Total: } 4\end{array}$ & Australia & $\begin{array}{l}\text { Examined the } \\
\text { differences in suicide } \\
\text { rates between urban } \\
\text { and rural areas of } \\
\text { Australia among } \\
\text { immigrants. } \\
\text { Population-study. }\end{array}$ & - & - & - & $\begin{array}{l}\text { Epidemiological data on the } \\
\text { entire Australia population. }\end{array}$ & $\begin{array}{l}\text { The higher rates of suicide in } \\
\text { older males in } \\
\text { non-metropolitan areas of } \\
\text { Australia is mainly due to the } \\
\text { high migrant suicide rates in } \\
\text { these regions, but the same it is } \\
\text { not true for the higher rates of } \\
\text { male youth suicide in } \\
\text { non-metropolitan areas. }\end{array}$ \\
\hline $\begin{array}{l}\text { Page et al., } 2006 \\
\text { [64] }\end{array}$ & $\begin{array}{l}\text { I:0 } \\
\text { II:1 } \\
\text { III:0 } \\
\text { IV:2 } \\
\text { Total: } 3\end{array}$ & Australia & $\begin{array}{l}\text { Examined the } \\
\text { correlation between } \\
\text { trends in suicide and } \\
\text { socio-economic status } \\
\text { (SES) in Australia. } \\
\text { Cross-sectional survey. }\end{array}$ & - & - & - & $\begin{array}{l}\text { Data from the Australian } \\
\text { Bureau of Statistics (ABS) }\end{array}$ & $\begin{array}{l}\text { Socio economic status (SES) } \\
\text { influenced suicide rates in } \\
\text { Australia and the suicide rate } \\
\text { was higher in low-SES males. } \\
\text { The suicide rate was lower in } \\
\text { young males in middle and } \\
\text { high SES groups. }\end{array}$ \\
\hline Law, 2014 [26] & $\begin{array}{l}\text { I:2 } \\
\text { II:1 } \\
\text { III:1 } \\
\text { IV:2 } \\
\text { Total: } 6\end{array}$ & Australia & $\begin{array}{l}\text { Investigated suicide } \\
\text { rates in } \\
\text { second-generation } \\
\text { migrants in Australia. } \\
\text { Cross-sectional survey. }\end{array}$ & 5541 & - & 5541 & $\begin{array}{l}\text { Data obtained from the } \\
\text { National Coroners' } \\
\text { Information System (NCIS). }\end{array}$ & $\begin{array}{l}\text { Second-generation migrants in } \\
\text { Australia had a lower suicide } \\
\text { risk compared to } \\
\text { first-generation migrants or } \\
\text { locals (third-plus-generation), } \\
\text { and this evidence could be } \\
\text { explained by their better } \\
\text { socioeconomic status. }\end{array}$ \\
\hline
\end{tabular}


Table 1. Cont.

\begin{tabular}{|c|c|c|c|c|c|c|c|c|}
\hline Study & Quality Score & Country & Aim and Study Design & Sample & Attempt $N$ & Suicide $N$ & Methods & Main Findings \\
\hline $\begin{array}{l}\text { Ide et al., } 2012 \\
\text { [65] }\end{array}$ & $\begin{array}{l}\text { I:3 } \\
\text { II:2 } \\
\text { III:0 } \\
\text { IV:2 } \\
\text { Total: } 7\end{array}$ & Australia & $\begin{array}{l}\text { Investigated suicide of } \\
\text { first-generation } \\
\text { immigrants in Australia } \\
\text { between } 1974 \text { and } 2006 . \\
\text { Epidemiological study. }\end{array}$ & - & - & - & $\begin{array}{l}\text { Data from Australian Institute } \\
\text { of Health and Welfare (AIHW). }\end{array}$ & $\begin{array}{l}\text { First-generation male migrants } \\
\text { in Australia had suicide rates } \\
\text { that correlated to those of their } \\
\text { countries of birth (COB), but } \\
\text { not females. Rates are probably } \\
\text { influenced by cultures, } \\
\text { traditions, ethnicities, and the } \\
\text { genetic predispositions of their } \\
\text { home country. All COB groups } \\
\text { showed suicide rates } \\
\text { decreasing in time. }\end{array}$ \\
\hline $\begin{array}{l}\text { Lipsicas et al., } \\
2012 \text { [66] }\end{array}$ & $\begin{array}{l}\text { I:3 } \\
\text { II:1 } \\
\text { III:0 } \\
\text { IV:2 } \\
\text { Total: } 6\end{array}$ & $\mathrm{EU}$ & $\begin{array}{l}\text { Investigated suicide } \\
\text { among immigrants in } \\
\text { European countries. } \\
\text { Cross-sectional survey. }\end{array}$ & 27,048 & - & - & $\begin{array}{l}\text { Data from the WHO/EURO } \\
\text { Multicentre Study on Suicidal } \\
\text { Behaviour }\end{array}$ & $\begin{array}{l}\text { Immigrants in European } \\
\text { countries showed significantly } \\
\text { higher suicide-attempt rates } \\
\text { (SARs) than their hosts and } \\
\text { had similar rates across } \\
\text { different European countries as } \\
\text { well as country-of-origin } \\
\text { suicide rate. }\end{array}$ \\
\hline $\begin{array}{l}\text { Loh et al., } 2007 \\
\text { [67] }\end{array}$ & $\begin{array}{l}\text { I:1 } \\
\text { II:1 } \\
\text { III:1 } \\
\text { IV:2 } \\
\text { Total: } 6\end{array}$ & Singapore & $\begin{array}{l}\text { Analysed suicide in } \\
\text { Singapore. } \\
\text { Cross-sectional survey. }\end{array}$ & 640 & - & 640 & $\begin{array}{l}\text { Data from the Center for } \\
\text { Forensic Medicine database. }\end{array}$ & $\begin{array}{l}\text { The suicide patterns in } \\
\text { Singapore showed a few } \\
\text { changes with the passing of } \\
\text { time. The characteristics that } \\
\text { remained constant for suicides } \\
\text { were: low prevalence for } \\
\text { teenagers, higher prevalence } \\
\text { for older adults, male/female } \\
\text { ratio, and method of jumping } \\
\text { from heights. Factors with } \\
\text { increased prevalence were: } \\
\text { unemployment and history of } \\
\text { psychiatric disorder. }\end{array}$ \\
\hline Lester, 1995 [68] & $\begin{array}{l}\text { I:0 } \\
\text { II:1 } \\
\text { III:0 } \\
\text { IV:2 } \\
\text { Total: } 3\end{array}$ & USA & Retrospective. & & & & $\begin{array}{l}\text { Data obtained from Kramer et } \\
\text { al. Predictor variables obtained } \\
\text { from the Census Bureau. }\end{array}$ & $\begin{array}{l}\text { The suicide rate of those born } \\
\text { in non-contiguous states of } \\
\text { America and abroad is } \\
\text { predicted by social } \\
\text { characteristics. }\end{array}$ \\
\hline $\begin{array}{l}\text { Kandrychyn, } \\
2004 \text { [69] }\end{array}$ & $\begin{array}{l}\text { I:0 } \\
\text { II:1 } \\
\text { III:0 } \\
\text { IV:2 } \\
\text { Total: } 3\end{array}$ & Russia & $\begin{array}{l}\text { Investigated how } \\
\text { suicide rates vary in } \\
\text { each geographic area } \\
\text { due to social factors, } \\
\text { migration, and } \\
\text { ethnicity. Retrospective. }\end{array}$ & - & - & - & $\begin{array}{l}\text { Data from the publication of } \\
\text { Goskomstat-the Russian State } \\
\text { Statistics Committee. }\end{array}$ & $\begin{array}{l}\text { The suicide rates vary through } \\
\text { Russian Federation and are } \\
\text { higher in the north because of } \\
\text { the historical prevalence of the } \\
\text { Finno-Ugrian component in } \\
\text { the north of the country. }\end{array}$ \\
\hline
\end{tabular}


Table 1. Cont.

\begin{tabular}{|c|c|c|c|c|c|c|c|c|}
\hline Study & Quality Score & Country & Aim and Study Design & Sample & Attempt $N$ & Suicide $N$ & Methods & Main Findings \\
\hline $\begin{array}{l}\text { Greenfield, } 2006 \\
\text { [70] }\end{array}$ & $\begin{array}{l}\text { I: } 1 \\
\text { II:2 } \\
\text { III: } 1 \\
\text { IV:1 } \\
\text { Total: } 5\end{array}$ & USA & $\begin{array}{l}\text { Examine suicide among } \\
\text { North American } \\
\text { adolescent immigrant } \\
\text { population. } \\
\text { Prospective. }\end{array}$ & 344 & 344 & - & Questionnaire & $\begin{array}{l}\text { Canadian immigrant } \\
\text { adolescents presented lower } \\
\text { suicide rates compared to } \\
\text { non-migrant ones, and this is } \\
\text { due to a lower rate of reported } \\
\text { drug use among immigrants. }\end{array}$ \\
\hline $\begin{array}{l}\text { Kwan et al., } \\
2007 \text { [20] }\end{array}$ & $\begin{array}{l}\text { I: } 2 \\
\text { II:1 } \\
\text { III:0 } \\
\text { IV:2 } \\
\text { Total: } 5\end{array}$ & China & $\begin{array}{l}\text { Examined suicide } \\
\text { among Hong Kong } \\
\text { adolescents. } \\
\text { Cross-sectional survey. }\end{array}$ & 4540 & - & - & $\begin{array}{l}\text { Questionnaire. Data from } \\
\text { Census \& Statistics } \\
\text { Department. }\end{array}$ & $\begin{array}{l}\text { This study indicated that } \\
\text { suicide rates among immigrant } \\
\text { adolescents in Hong Kong } \\
\text { depend on duration of } \\
\text { residence: short-duration }(<10 \\
\text { years) correlated with lower } \\
\text { suicide rates, and } \\
\text { long-duration }(>10 \text { years or } \\
\text { more) with higher suicide rates } \\
\text { than the local-born } \\
\text { counterparts. }\end{array}$ \\
\hline $\begin{array}{l}\text { Malenfant et al., } \\
2004 \text { [71] }\end{array}$ & $\begin{array}{l}\text { I:0 } \\
\text { II:1 } \\
\text { III:0 } \\
\text { IV:2 } \\
\text { Total: } 3\end{array}$ & Canada & $\begin{array}{l}\text { Examined suicide } \\
\text { among Canadian } \\
\text { immigrants. } \\
\text { Cross-sectional survey. }\end{array}$ & - & - & - & $\begin{array}{l}\text { Data from the Canadian Vital } \\
\text { Statistics Data Base and the } \\
\text { World Health Statistics Annual } \\
\text { of the World Health } \\
\text { Organization. }\end{array}$ & $\begin{array}{l}\text { This study examined } \\
\text { differences in suicide rates } \\
\text { between immigrants and } \\
\text { non-immigrant Canadian } \\
\text { residents. Immigrants had } 50 \% \\
\text { lower suicide rates, which } \\
\text { increased with age and } \\
\text { involved predominately males. }\end{array}$ \\
\hline
\end{tabular}




\subsection{Suicidal Ideation and Attempt among Ethnic Minorities}

Studies have shown higher rates of suicidal behaviour and self-harm in ethnic minorities. O'Keefe and colleagues reported that American Indian suicidal ideation can be predicted by thwarted belongingness and perceived burdensomeness [40]. The study involved 171 American Indians (representative of 27 different tribes) and employed an online survey based on an empirically supported theoretical model of suicide-the Interpersonal-Psychological Theory of Suicide [72]. The results showed that the interaction of thwarted belongingness and perceived burdensomeness predicted suicidal ideation better than each predictor taken individually [40].

Walker and colleagues investigated the role of acculturation in suicidal-behaviour among African descendants living in the USA, in a sample of 423 adults using the African American Acculturation Scale. Contrary to previous studies, it was found that just religious well-being, and not acculturation, was predictive of suicidal ideation and history of suicide attempt [46].

Else and colleagues identified, among Hawaiian youth, high levels of acculturation as a risk factor for suicide attempts [73]. Moreover, Scheel examined suicidal behaviour among American Indian college students [41]. They found that American Indian college students' suicidality was characterized by: a rate of suicidal ideation comparable to general college students and low rates of awareness of traditional tribal suicide ( $10 \%)$. Moreover, $57 \%$ of the participants with suicidal ideation reported that they would not seek help from a mental health professional. Furthermore, they identified that the help-seeking likelihood depended on the cultural commitment. Participants more committed to tribal culture prefer counselling from American Indian counsellors (e.g., tribal healer/medicine man: 22\%; American Indian mental health professional from university: $24 \%$; from the campus: $44 \%$; outside the campus: $41 \%$ ). Less committed participants instead showed a moderate openness to seeking help from non-American Indian counsellors [41].

\subsection{Suicide Deaths in Ethnic Minorities}

Studies have shown increasing rates of suicide deaths, especially in youths from ethnic minorities [42,73]. In Native Hawaiians, death by suicide is a relatively frequent phenomenon among adolescents and young adults, as they have higher lifetime prevalence rates of suicide attempts $(12.9 \%)$ in comparison with non-Hawaiian students (9.6\%) [73].

On the other hand, among the ethnic minorities living in the United Kingdom, black Caribbean $(\mathrm{SMR}=0.26)$ and South Asian (SMR $=0.4)$ women showed a lower risk of suicide compared to the native population [44]. Black Caribbean and young Black African men (SMR $=2.05)$ were revealed to have, instead, a higher risk of suicide than the native population [44]. In Hawaiians and other Pacific Islander populations, rates and distribution of suicide deaths were similar to other indigenous population (such as Maori in New Zealand), with a first peak in adolescents and young adults (annual suicide mortality rates per 100,000 by age: $52.8 / 100,000$ for $15-24$-year-olds, increasing to $72.4 / 100,000$ for 25-44-year-olds) and then a sharp decline instead of a second peak in the elderly, which is typical of the bimodal distribution in United States and most Western countries [73].

Wong and colleagues focused on youth risk factors for suicide in ethnic minorities among American high school students, using data from the 1999-2009 Youth Risk Behaviour Surveys [42]. Native Hawaiian/Pacific Islander adolescents and multiracial adolescents had a higher prevalence of risk factors for suicide (such as depression, suicide ideation, plans, attempts and /or severe attempts), comparable to that of American Indian/Alaska Native adolescents already known in the literature as an "at-risk population" [42].

Bhui and colleagues also investigated the possible role of ethnicity in suicidal behaviour and related risk indicators among patients within a year of contact with psychiatric services [39,44]. Although they revealed that ethnicity influenced suicide rates and indicators of suicide risk, classical indicators of suicide risk such as "risk-related symptoms" (suicidal ideas, depressive symptoms, emotional distress, and hopelessness) were less common in ethnic groups such as black Africans (SMR 2.05) and South Asians, as compared to white British individuals [44]. Another study 
conducted by Ngwena in London public health services focused on trends of suicidal behaviour in black and minority ethnic (BME) groups. Among 996 BME patients admitted to the acute psychiatric ward with suicide attempt or self-harm-injury, those of Arab origin and South Americans were more prevalent (28\%), followed by Western and Eastern Europeans (26\%) [45].

Hunt identified the characteristics of psychiatric patients from ethnic minorities who died by suicide through the observation of 282 suicidal patients from ethnic minorities (6\% of total) who had been in contact with mental health services in the 12 months prior to death. In these patients, suicide was characterized by: more violent methods, first episode of self-harm, higher rates of schizophrenia, unemployment, history of violence, and drug misuse [43].

\subsection{Cultural Stress as Risk Factor for Suicidal Behaviour in Immigrants and Ethnic Minorities}

Hagaman and colleagues investigated suicides among Bhutanese refugees resettled in the United States between 2008 and 2011, attempting to identify psychological characteristics and risk factors. Considering the small sample size, they showed a large presence of different post-migration difficulties in the investigated population [48]. The most common risk factor was a language barrier $(71 \%)$, which seems to contribute to the development of hopelessness. Separation from family (43\%) and worrying about family back home $(57 \%)$ were also recognized as contributing factors to suicidal behaviour. Ngwena suggested other risk factors for self-harm, suicide, or suicide attempt in black and minority ethnic groups: arranged/forced marriage, lack of information on the health care system, loss of status and loss of social network, and also acculturation and thwarted ambition [45]. High levels of acculturation was identified as a risk factor for Hawaiian youth suicide attempts in another study [73]. In African descendants living in the USA, religious well-being was found to be a predictor for suicidal ideation [46].

Wong confirmed that multiracial adolescents more frequently reported a liability to mental disorders and health risk behaviours or dangerous conducts, such as substance use and violence [42]. In addition, immigrant status contributed negatively to acculturative stress, cultural conflicts, and socioeconomic difficulties. In England and Wales, as compared to native white people, suicidal ideation, emotional distress, and hostility were found to be less frequent among black Africans, black Caribbean individuals, and South Asians, while depression and a sense of hopelessness were more prevalent among South Asians [39]. These differences between native people and minorities suggest potentially different pathways to suicidal behaviour among different populations and that cultural stressors play a key role as risk factors for suicidal behaviour.

\section{Discussion}

The present overview suggested that findings on suicidal behaviour in immigrants are complex and it is difficult to delineate a theoretical framework explaining findings from the literature. On one hand, several studies found higher rates of suicide attempts among immigrants compared to native populations and that immigrants have a higher risk of experiencing suicidal behaviour than the same population in their home countries [27-29,74]. On the other hand, some studies did not reveal differences in suicidal behaviour between immigrant and native populations [22]. However, among the immigrant group and ethnic minorities, specific populations should be considered with regard to their risk of attempting suicide and also for suicide deaths, such as South Asian and black African women [36,39]. In terms of the literature related to immigrants, we found a lack of studies related to suicidal behaviour in ethnic minorities. The highest risk of suicide was found among black Caribbean individuals and young black African men living in the United Kingdom, suggesting that this might be related to specific environmental and psychosocial risk factors [44]. Some authors focused specifically on Native Hawaiians [73] and American Indian/Alaska Native adolescents [42], which have been found to be populations at risk for suicide death.

Various studies have also highlighted that immigrants and ethnic minorities do not receive the same psychiatric care during or after a suicide attempt or are less likely to contact psychiatric services 
when experiencing suicidal thoughts and/or engaging in suicidal behaviour $[33,39,43]$. This may lead to a global worsening of their mental health condition and an increased risk of suicide. In addition, if we take into consideration the different rates of suicide attempts and deaths, evidence indicates various patterns and mechanisms.

According to the Interpersonal Psychological Theory of suicide, perceived burdensomeness is a key ingredient for a heightened risk of suicide, together with low belonging, social alienation and the ability to enact self-injury behaviours [72]. Although Joiner's theory holds true for non-minorities and non-immigrants as well, it can provide an explanation of the underlying mechanism related to the increased risk of suicide presented by these populations. Indeed, socioeconomic factors such as poor socio-economic status, social exclusion, discrimination, or deprivation $[45,75]$ are more clearly related to suicidal behaviour rather than the status of migrant itself $[25,64]$. In conclusion, our findings suggest that migrants and ethnic minorities may be considered as a moderate-high risk group for suicidal behaviour, but this requires further and more specific investigation. Interestingly, ethnicity is related to different risk factors for suicide that are common among general population, such as; suicidal ideas, depressive symptoms, emotional distress, and hopelessness. These risk factors are less common in ethnic groups such as black Africans and South Asians [44]. This suggests that ethnic minorities may manifest a specific phenotype of suicidal behaviour; however, more studies are needed to understand the underlying mechanisms that may explain these differences. Further studies are needed to develop specific suicide prevention strategies targeting ethnic minorities, which represent an effective public health intervention.

\section{Limitations}

The present review should be considered in the light of several limitations. First, the articles included in our synthesis were significantly different in terms of sample size, study design, and methodology, and this made it difficult to provide a quantitative synthesis. In addition, the samples included in this review varied widely on age, instruction, culture, traditions, and religious beliefs. Future studies should focus on investigating different rates and patterns of suicidal behaviour in the same population of migrants or ethnic minority longitudinally, in order to evaluate the influence of integration and acculturation on reducing suicidal behaviour.

\section{Conclusions}

Taken together, a majority of studies demonstrated higher rates of suicide attempts among immigrants than the native population. Risk factors among migrants and ethnic minorities were found to be: language barriers, worrying about family back home, and separation from family. Lack of information on the health care system, loss of status, loss of social network, and acculturation were identified as possible triggers for suicidal behaviour. Both migrant populations and ethnic minorities may have unique risk factors for suicidal behaviour; however, more studies are needed to clarify them. Additional research is required in order to develop a more reliable and standardized assessment of suicidal behaviour in these populations and to identify dedicated strategies of risk prevention for each group. It is also relevant for future studies to address the specific factors that influence suicidal thoughts and behaviours in each different ethnic group.

Author Contributions: Conceptualization, A.F. (Alberto Forte) and M.P.; Methodology, A.F. (Alberto Forte), F.G., F.T.; Software, A.F. (Alberto Forte), F.G., F.T.; Validation, A.F. (Alberto Forte) and M.P.; Formal Analysis, A.F. (Alberto Forte); Investigation, A.F. (Alberto Forte), F.G., F.T.; Resources, A.F. (Alberto Forte), F.G., F.T., M.P.; Data Curation, A.F. (Alberto Forte), F.G., F.T., M.P.; Writing-Original Draft Preparation, A.F. (Alberto Forte), M.P., D.A.L.; Writing-Review \& Editing, A.F. (Alberto Forte), M.P., D.A.L.; Visualization, G.C., V.G., A.F. (Andrea Fiorillo); Supervision and Validation, M.P., P.G., please turn to the CRediT taxonomy for the term explanation. Authorship must be limited to those who have contributed substantially to the work reported.

Funding: No author or immediate family member has financial relationships with commercial entities that might represent the appearance of a potential conflict of interest.

Conflicts of Interest: The authors declare no conflict of interest. 


\section{References}

1. Abbott, A. The mental-health crisis among migrants. Nature 2016, 538, 158-160. [CrossRef] [PubMed]

2. Carta, M.G.; Bernal, M.; Hardoy, M.C.; Haro-Abad, J.M.; The "Report on the Mental Health in Europe" Working Group. Migration and mental health in Europe (the state of the mental health in Europe working group: Appendix 1). Clin. Pract. Epidemiol. Ment. Health 2005, 1, 13. [CrossRef] [PubMed]

3. Straiton, M.; Reneflot, A.; Diaz, E. Immigrants' use of primary health care services for mental health problems. BMC Health Serv. Res. 2014, 14, 341. [CrossRef] [PubMed]

4. Derr, A.S. Mental Health Service Use among Immigrants in the United States: A Systematic Review. Psychiatr. Serv. 2016, 67, 265-274. [CrossRef] [PubMed]

5. Bhugra, D.; Gupta, S.; Bhui, K.; Craig, T.; Dogra, N.; Ingleby, J.D.; Kirkbride, J.; Moussaoui, D.; Nazroo, J.; Qureshi, A.; et al. WPA guidance on mental health and mental health care in migrants. World Psychiatry 2011, 10, 2-10. [CrossRef] [PubMed]

6. Gilliver, S.C.; Sundquist, J.; Li, X.; Sundquist, K. Recent research on the mental health of immigrants to Sweden: A literature review. Eur. J. Public Health 2014, 24, 72-79. [CrossRef] [PubMed]

7. Lindert, J.; Ehrenstein, O.S.; Priebe, S.; Mielck, A.; Brähler, E. Depression and anxiety in labor migrants and refugees-A systematic review and meta-analysis. Soc. Sci. Med. 2009, 69, 246-257. [CrossRef] [PubMed]

8. Cantor-Graae, E.; Selten, J.-P. Schizophrenia and Migration: A Meta-Analysis and Review. Am. J. Psychiatry 2005, 162, 12-24. [CrossRef] [PubMed]

9. Falah-Hassani, K.; Shiri, R.; Vigod, S.; Dennis, C.-L. Prevalence of postpartum depression among immigrant women: A systematic review and meta-analysis. J. Psychiatr. Res. 2015, 70, 67-82. [CrossRef] [PubMed]

10. Spallek, J.; Reeske, A.; Norredam, M.; Nielsen, S.S.; Lehnhardt, J.; Razum, O. Suicide among immigrants in Europe-A systematic literature review. Eur. J. Public Health 2015, 25, 63-71. [CrossRef] [PubMed]

11. Heredia Montesinos, A. Precipitating and risk factors for suicidal behavior among immigrants and ethnic minorities in Europe: A review of the literature. Suicidal Behav. Immigrants Ethn. Minor. Eur. 2015, 4, 60-80.

12. Al-Sharifi, A.; Krynicki, C.R.; Upthegrove, R. Self-harm and ethnicity: A systematic review. Int. J. Soc. Psychiatry 2015, 61, 600-612. [CrossRef] [PubMed]

13. Wyatt, L.C.; Ung, T.; Park, R.; Kwon, S.C.; Trinh-Shevrin, C. Risk Factors of Suicide and Depression among Asian American, Native Hawaiian, and Pacific Islander Youth: A Systematic Literature Review. J. Health Care Poor Underserved 2015, 26, 191-237. [CrossRef] [PubMed]

14. Abraído-Lanza, A.F.; Armbrister, A.N.; Flórez, K.R.; Aguirre, A.N. Toward a theory-driven model of acculturation in public health research. Am. J. Public Health 2006, 96, 1342-1346. [CrossRef] [PubMed]

15. Williams, C.L.; Berry, J.W. Primary prevention of acculturative stress among refugees. Application of psychological theory and practice. Am. Psychol. 1991, 46, 632-641. [CrossRef] [PubMed]

16. Liberati, A.; Altman, D.G.; Tetzlaff, J.; Mulrow, C.; Gøtzsche, P.C.; Ioannidis, J.P.A.; Clarke, M.; Devereaux, P.J.; Kleijnen, J.; Moher, D. The PRISMA statement for reporting systematic reviews and meta-analyses of studies that evaluate health care interventions: Explanation and elaboration. Res. Methods Rep. 2009, 339. [CrossRef]

17. Simon, R.I.; Hales, R.E.; American Psychiatric Publishing. The American Psychiatric Publishing Textbook of Suicide Assessment and Management; American Psychiatric Pub: Philadelphia, PA, USA, 2012.

18. UNHCR General Assembly Statute of the Office of the United Nations High Commissioner for RefugeesStatute of the Office of the United Nations High Commissioner for Refugees 428 (V). Available online: http://www.unhcr.org/excom/bgares/3ae69ee64/statute-office-united-nations-highcommissioner-refugees.html (accessed on 6 July 2018).

19. Šmihula, D. National minorities in the law of the EC/EU. Rom. J. Eur. Aff. 2008, 8, 51-58.

20. Kwan, Y.K.; Ip, W.C. Suicidality and migration among adolescents in Hong Kong. Death Stud. 2007, 31, 45-66. [CrossRef] [PubMed]

21. Dai, J.; Zhong, B.L.; Xiang, Y.T.; Chiu, H.F.K.; Chan, S.S.M.; Yu, X.; Caine, E.D. Internal migration, mental health, and suicidal behaviors in young rural Chinese. Soc. Psychiatry Psychiatr. Epidemiol. 2015, 50, 621-631. [CrossRef] [PubMed]

22. Shoval, G.; Schoen, G.; Vardi, N.; Zalsman, G. Suicide in Ethiopian immigrants in Israel: A case for study of the genetic-environmental relation in suicide. Arch. Suicide Res. 2007, 11, 247-253. [CrossRef] [PubMed] 
23. van Bergen, D.D.; Eikelenboom, M.; Smit, J.H.; van de Looij-Jansen, P.M.; Saharso, S. Suicidal behavior and ethnicity of young females in Rotterdam, The Netherlands: Rates and risk factors. Ethn. Health 2010, 15, 515-530. [CrossRef] [PubMed]

24. Termorshuizen, F.; Wierdsma, A.I.; Visser, E.; Drukker, M.; Sytema, S.; Laan, W.; Smeets, H.M.; Selten, J.P. Psychosis and suicide risk by ethnic origin and history of migration in the Netherlands. Schizophr. Res. 2012, 138, 268-273. [CrossRef] [PubMed]

25. Di Thiene, D.; Alexanderson, K.; Tinghög, P.; La Torre, G.; Mittendorfer-Rutz, E. Suicide among First-generation and Second-generation Immigrants in Sweden: Association with Labour Market Marginalisation and Morbidity. J. Epidemiol. Community Health 2015, 69, 467-473. [CrossRef] [PubMed]

26. Law, C.K.; Kolves, K.; De Leo, D. Suicide mortality in second-generation migrants, Australia, 2001-2008. Soc. Psychiatry Psychiatr. Epidemiol. 2014, 49, 601-608. [CrossRef] [PubMed]

27. Kposowa, A.J.; McElvain, J.P.; Breault, K.D. Immigration and Suicide: The Role of Marital Status, Duration of Residence, and Social Integration. Arch. Suicide Res. 2008, 12, 82-92. [CrossRef] [PubMed]

28. Al-Maskari, F.; Shah, S.M.; Al-Sharhan, R.; Al-Haj, E.; Al-Kaabi, K.; Khonji, D.; Schneider, J.D.; Nagelkerke, N.J.; Bernsen, R.M. Prevalence of depression and suicidal behaviors among male migrant workers in United Arab Emirates. J. Immigr. Minor. Health 2011, 13, 1027-1032. [CrossRef] [PubMed]

29. Lipsicas, C.B.; Mäkinen, I.H.; Wasserman, D.; Apter, A.; Kerkhof, A.; Michel, K.; Renberg, E.S.; Van Heeringen, K.; Värnik, A.; Schmidtke, A. Repetition of attempted suicide among immigrants in Europe. Can. J. Psychiatry 2014, 59, 539-547. [CrossRef] [PubMed]

30. Johansson, L.M.; Sundquist, J.; Johansson, S.E.; Qvist, J.; Bergman, B. The influence of ethnicity and social and demographic factors on Swedish suicide rates. A four year follow-up study. Soc. Psychiatry Psychiatr. Epidemiol. 1997, 32, 165-170. [PubMed]

31. Ryan, L.; Leavey, G.; Golden, A.; Blizard, R.; King, M. Depression in Irish migrants living in London: Case-control study. Br. J. Psychiatry 2006, 188, 560-566. [CrossRef] [PubMed]

32. Leavey, G. Suicide and Irish migrants in Britain: Identity and integration. Int. Rev. Psychiatry 1999, 11, 168-172. [CrossRef]

33. Ayalon, L. Suicidal and Depressive Symptoms in Filipino Home Care Workers in Israel. J. Cross. Cult. Gerontol. 2012, 27, 51-63. [CrossRef] [PubMed]

34. Burger, I.; Van Hemert, A.M.; Schudel, W.J.; Middelkoop, B.J.C. Suicidal Behavior in Four Ethnic Groups in the Hague, 2002-2004. Crisis 2009, 30, 63-67. [CrossRef] [PubMed]

35. Duldulao, A.A.; Takeuchi, D.; Hong, S. Correlates of Suicidal Behaviors among Asian Americans. Arch. Suicide Res. 2009, 13, 277-290. [CrossRef] [PubMed]

36. Bhugra, D. Suicidal behavior in South Asians in the UK. Crisis 2002, 23, 108-113. [CrossRef] [PubMed]

37. Wong, Y.J.; Vaughan, E.L.; Liu, T.; Chang, T.K. Asian Americans' proportion of life in the United States and suicide ideation: The moderating effects of ethnic subgroups. Asian Am. J. Psychol. 2014, 5, 237-242. [CrossRef]

38. Shah, A.; Lindesay, J.; Dennis, M. Suicides by country of birth groupings in England and Wales: Age-associated trends and standardised mortality ratios. Soc. Psychiatry Psychiatr. Epidemiol. 2011, 46, 197-206. [CrossRef] [PubMed]

39. Bhui, K.S.; McKenzie, K. Rates and risk factors by ethnic group for suicides within a year of contact with mental health services in England and Wales. Psychiatr. Serv. 2008, 59, 414-420. [CrossRef] [PubMed]

40. O'Keefe, V.M.; Wingate, L.R.; Tucker, R.P.; Rhoades-Kerswill, S.; Slish, M.L.; Davidson, C.L. Interpersonal suicide risk for American Indians: Investigating thwarted belongingness and perceived burdensomeness. Cult. Divers. Ethn. Minor. Psychol. 2014, 20, 61-67. [CrossRef] [PubMed]

41. Scheel, K.R.; Prieto, L.R.; Biermann, J. American Indian college student suicide: Risk, beliefs, and help-seeking preferences. Couns. Psychol. Q. 2011, 24, 277-289. [CrossRef]

42. Wong, S.S.; Sugimoto-Matsuda, J.J.; Chang, J.Y.; Hishinuma, E.S. Ethnic differences in risk factors for suicide among American high school students, 2009: The vulnerability of multiracial and Pacific Islander adolescents. Arch. Suicide Res. 2012, 16, 159-173. [CrossRef] [PubMed]

43. Hunt, I.M.; Robinson, J.; Bickley, H.; Meehan, J.; Parsons, R.; McCann, K.; Flynn, S.; Burns, J.; Shaw, J.; Kapur, N.; et al. Suicides in ethnic minorities within 12 months of contact with mental health services. National clinical survey. Br. J. Psychiatry 2003, 183, 155-160. [CrossRef] [PubMed] 
44. Bhui, K.S.; Dinos, S.; McKenzie, K. Ethnicity and its influence on suicide rates and risk. Ethn. Health 2012, 17, 141-148. [CrossRef] [PubMed]

45. Ngwena, J. Black and minority ethnic groups (BME) suicide, admission with suicide or self-harm: An inner city study. J. Public Health 2014, 22, 155-163. [CrossRef]

46. Walker, R.L.; Utsey, S.O.; Bolden, M.A.; Williams, O. Do sociocultural factors predict suicidality among persons of African descent living in the U.S.? Arch. Suicide Res. 2005, 9, 203-217. [CrossRef] [PubMed]

47. Chung, I.W.; Caine, E.D.; Barron, C.T.; Badaracco, M.A. Clinical and psychosocial profiles of Asian immigrants who repeatedly attempt suicide. Crisis 2015, 36, 353-362. [CrossRef] [PubMed]

48. Hagaman, A.K.; Sivilli, T.I.; Ao, T.; Blanton, C.; Ellis, H.; Lopes Cardozo, B.; Shetty, S. An Investigation into Suicides Among Bhutanese Refugees Resettled in the United States Between 2008 and 2011. J. Immigr. Minor. Health 2016, 18, 19-27. [CrossRef] [PubMed]

49. Eaton, D.K.; Foti, K.; Brener, N.D.; Crosby, A.E.; Flores, G.; Kann, L. Associations between risk behaviors and suicidal ideation and suicide attempts: Do racial/ethnic variations in associations account for increased risk of suicidal behaviors among Hispanic/Latina 9th- to 12th-grade female students? Arch. Suicide Res. 2011, 15, 113-126. [CrossRef] [PubMed]

50. Peña, J.B.; Wyman, P.A.; Brown, C.H.; Matthieu, M.M.; Olivares, T.E.; Hartel, D.; Zayas, L.H. Immigration generation status and its association with suicide attempts, substance use, and depressive symptoms among Latino adolescents in the USA. Prev. Sci. 2008, 9, 299-310. [CrossRef] [PubMed]

51. Hovey, J.D.; Magaña, C.G. Suicide Risk Factors among Mexican Migrant Farmworker Women in the Midwest United States. Arch. Suicide Res. 2003, 7, 107-121. [CrossRef]

52. Borges, G.; Breslau, J.; Su, M.; Miller, M.; Medina-Mora, M.E.; Aguilar-Gaxiola, S. Immigration and suicidal behavior among Mexicans and Mexican Americans. Am. J. Public Health 2009, 99, 728-733. [CrossRef] [PubMed]

53. Sorenson, S.B.; Shen, H. Mortality among young immigrants to California: Injury compared to disease deaths. J. Immigr. Health 1999, 1, 41-47. [CrossRef] [PubMed]

54. Walsh, S.D.; Edelstein, A.; Vota, D. Suicidal ideation and alcohol use among ethiopian adolescents in Israel the relationshipwith ethnic identity and parental support. Eur. Psychol. 2012, 17, 131-142. [CrossRef]

55. Aichberger, M.C.; Heredia Montesinos, A.; Bromand, Z.; Yesil, R.; Temur-Erman, S.; Rapp, M.A.; Heinz, A.; Schouler-Ocak, M. Suicide attempt rates and intervention effects in women of Turkish origin in Berlin. Eur. Psychiatry 2015, 30, 480-485. [CrossRef] [PubMed]

56. Voracek, M.; Loibl, L.M.; Dervic, K.; Kapusta, N.D.; Niederkrotenthaler, T.; Sonneck, G. Consistency of immigrant suicide rates in Austria with country-of-birth suicide rates: A role for genetic risk factors for suicide? Psychiatry Res. 2009, 170, 286-289. [CrossRef] [PubMed]

57. Johansson, L.M.; Sundquist, J.; Johansson, S.E.; Bergman, B.; Qvist, J.; Träskman-Bendz, L. Suicide among foreign-born minorities and native swedes: An epidemiological follow-up study of a defined population. Soc. Sci. Med. 1997, 44, 181-187. [CrossRef]

58. Stack, S. The Effect of Immigration on Suicide: A Cross-National Analysis. Basic Appl. Soc. Psych. 1981, 2, 205-218. [CrossRef]

59. Burvill, P.W. Migrant suicide rates in Australia and in country of birth. Psychol. Med. 1998, 28, $201-208$. [CrossRef] [PubMed]

60. Värnik, A.; Kõlves, K.; Wasserman, D. Suicide among Russians in Estonia: Database study before and after independence. BMJ 2005, 330, 176-177. [CrossRef] [PubMed]

61. Maynard, M.J.; Rosato, M.; Teyhan, A.; Harding, S. Trends in suicide among migrants in England and Wales 1979-2003. Ethn. Health 2012, 17, 135-140. [CrossRef] [PubMed]

62. Taylor, R.; Morrell, S.; Slaytor, E.; Ford, P. Suicide in urban New South Wales, Australia 1985-1994: Socio-economic and migrant interactions. Soc. Sci. Med. 1998, 47, 1677-1686. [CrossRef]

63. Morrell, S.; Taylor, R.; Slaytor, E.; Ford, P. Urban and rural suicide differentials in migrants and the Australian-Born, New South Wales, Australia 1985-1994. Soc. Sci. Med. 1999, 49, 81-91. [CrossRef]

64. Page, A.; Morrell, S.; Taylor, R.; Carter, G.; Dudley, M. Divergent trends in suicide by socio-economic status in Australia. Soc. Psychiatry Psychiatr. Epidemiol. 2006, 41, 911-917. [CrossRef] [PubMed]

65. Ide, N.; Kõlves, K.; Cassaniti, M.; De Leo, D. Suicide of first-generation immigrants in Australia, 1974-2006. Soc. Psychiatry Psychiatr. Epidemiol. 2012, 47, 1917-1927. [CrossRef] [PubMed] 
66. Lipsicas, C.B.; Makinen, I.H.; Apter, A.; De Leo, D.; Kerkhof, A.; Lönnqvist, J.; Michel, K.; Renberg, E.S.; Sayil, I.; Schmidtke, A.; et al. Attempted suicide among immigrants in European countries: An international perspective. Soc. Psychiatry Psychiatr. Epidemiol. 2012, 47, 241-251. [CrossRef] [PubMed]

67. Loh, M.; Tan, C.H.; Sim, K.; Lau, G.; Mondry, A.; Leong, J.Y.; Tan, E.C. Epidemiology of completed suicides in Singapore for 2001 and 2002. Crisis 2007, 28, 148-155. [CrossRef] [PubMed]

68. Lester, D. Explaining regional differences in suicide rates. Soc. Sci. Med. 1995, 40, 719-721. [CrossRef]

69. Kandrychyn, S. Geographic variation in suicide rates: Relationships to social factors, migration, and ethnic history. Arch. Suicide Res. 2004, 8, 303-314. [CrossRef] [PubMed]

70. Greenfield, B.; Rousseau, C.; Slatkoff, J.; Lewkowski, M.; Davis, M.; Dube, S.; Lashley, M.E.; Morin, I.; Dray, P.; Harnden, B. Profile of a Metropolitan North American Immigrant Suicidal Adolescent Population. Can. J. Psychiatry 2006, 51, 155-159. [CrossRef] [PubMed]

71. Malenfant, E.C. Suicide in Canada's immigrant population. Health Rep. 2004, 15, 9-17. [PubMed]

72. Joiner, T.E. Why People Die by Suicide; Harvard University Press: Cambridge, MA, USA, 2005.

73. Else, I.R.N.; Andrade, N.N.; Nahulu, L.B. Suicide and suicidal-related behaviors among indigenous Pacific Islanders in the United States. Death Stud. 2007, 31, 479-501. [CrossRef] [PubMed]

74. Lipsicas, C.B.; Mäkinen, I.H. Immigration and suicidality in the young. Can. J. Psychiatry 2010, 55, $274-281$. [CrossRef] [PubMed]

75. Taylor, M.R.; Boden, J.M.; Rucklidge, J.J. The relationship between ADHD symptomatology and self-harm, suicidal ideation, and suicidal behaviours in adults: A pilot study. ADHD Atten. Deficit Hyperact. Disord. 2014, 6, 303-312. [CrossRef] [PubMed]

(C) 2018 by the authors. Licensee MDPI, Basel, Switzerland. This article is an open access article distributed under the terms and conditions of the Creative Commons Attribution (CC BY) license (http:/ / creativecommons.org/licenses/by/4.0/). 